\title{
Graphic Computerization of Cephalometric Data
}

\author{
RICHARD L. MILLER, DICK J. DIJKMAN, MICHAEL L. RIOLO, and \\ ROBERT E. MOYERS \\ Center for Human Growth and Development, The University of Michigan, Ann Arbor, \\ Michigan 48104, USA
}

A computer system for storage, retrieval, and analysis of cephalometric samples, developed at the Center for Human Growth and Development and reported previously (R. L. MLLER, W. S. Hunter, and R. E. MoYers, J Dent Res 49:1176, 1970), has been modified to improve collection speed and accuracy.

Tridea Computer Graphics, Inc.* has developed and manufactures an automated, computer-operated graphics system, designed to quickly turn line drawings into machine tool instructions for manufacturing. The device lends itself to the automatic collection of cephalometric line tracings. Cephalometric tracings consisting of anatomic contours (lines) are scanned by an automatic, computer-controlled, center of the line follower, the position of which provides $X$ and $Y$ coordinates for storage on magnetic or paper tape. A television view system with a $\times 10$ magnification and $a$ manual control allows the operator to position and monitor the line follower. The operator controls the sequencing of lines to be digitized, and manually aids the line follower where line quality or line intersections make automatic line following difficult. A special repositioning feature of the graphics system computer program permits correction of erroneous data before it appears on tape.

The software also allows a wide range of output formats and digitizing accuracy; the maximum resolution is $0.025 \mathrm{~mm}$. Another important software feature is the algorithm by which a point on a line is selected for inclusion in the output data. With this algorithm, curves of greater curvature have a greater number of points per unit length, and this results in improved resolution. Special console control may be used to flag certain points to indicate landmarks, end of lines, etc. The collection of a typical tracing of approximately 3,000 points requires ten minutes.

The Tridea Aldraft machine can verify the data taped by plotting the digitized outlines. This graphic representation of the data (Illustration) is produced on any desired medium such as vellum, nylon, a scribe coat, or metal plate in approximately five minutes. Accuracy

Supported by USPHS Grant No. HD-02272 from the National Institute of Dental Research, National Institutes of Health, Bethesda, Md.

Additional information available on request to authors.

Received for publication April 3, 1971.

* Actron Industries, Inc., 700 Royal Oaks Drive, Monrovia, Calif.

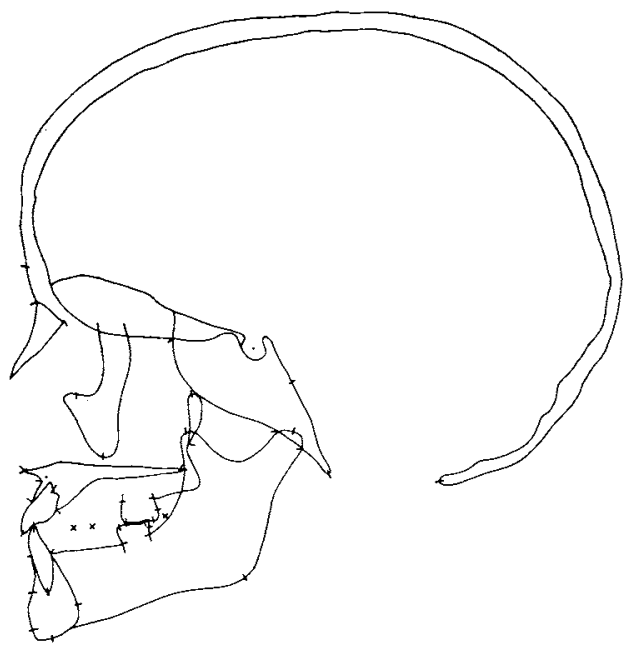

A Tridea verification plot illustrating data collected.

checks can then be made by superimposition of the original tracing on the plot.

Special software permits perspective views to be obtained from three-axis orthographic data. These three-dimensional models, as constructed in a plot or stored on data tape, are a unique feature compared to the two-dimensional graphics system previously reported.

The data are stored in a format that can be readily used for various software applications particular to the needs of investigators and may be used as input for further processing in any general purpose computer and particularly with the system already described.

This system uniquely allows the collection of high resolution, computer manipulative, morphologically and anatomically relevant data. The capability of this device to collect three-dimensional data from orthogonal, twodimensional tracings is a useful advance for investigators studying the growth and architecture of living structures. Furthermore, considerable time and cost savings can be realized in the use of this system compared with others surveyed.

We acknowledge the efforts of A. B. Coulter whose technical assistance as a Tridea representative made this design possible. 